\title{
Surgical treatment of a rare association: Aberrant right coronary origination, coronary arteriovenous fistula, and aortic root aneurysm
}

\author{
Frédéric Vanden Eynden, MD, ${ }^{a}$ Philippe Demers, MD, ${ }^{\mathrm{b}}$ Bertrand Marcheix, MD, ${ }^{\mathrm{b}}$ \\ Denis Bouchard, MD, ${ }^{\mathrm{b}}$ Raymond Cartier, MD, ${ }^{\mathrm{b}}$ and Michel Pellerin, MD, \\ Brussels, Belgium, and Montreal, Quebec, Canada
}

Supplemental material is available online.

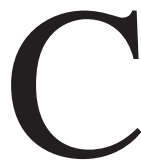

oronary artery anomalies and coronary artery fistulas are rare congenital entities that are not usually associated. These two abnormalities combined made it possible for a third unusual event, namely annuloaortic ectasia in a young patient without any feature of connective tissue disease, to induce right ventricular failure. A modified Bentall procedure with reimplantation of the left coronary artery on the anterior portion of the prosthesis solved the three problems and restored right ventricular function.

\section{Clinical Summary}

A 39-year-old man of Philippine origin was admitted to the emergency department for increasing dyspnea. He had no past medical history, except arterial hypertension treated with angiotensinconverting enzyme inhibitors. Two weeks before admission, he started complaining of shortness of breath for usual physical activities without any thoracic discomfort, cough, or fever. The physical examination was unremarkable except for hypertension and a diastolic heart murmur, which was increased with handgrip. Chest radiography was pertinent for mild pulmonary edema, and the patient was treated accordingly. A cardiac echocardiogram showed an enlarged aortic root, ascending aorta, and a dilated annulus at $31 \mathrm{~mm}$ with severe aortic insufficiency. There was severe left ventricular enlargement, as well as severe systolic left and right ventricular dysfunction (left ventricular ejection fraction, $32 \%$; right ventricular ejection fraction, $34 \%$ ). Coronary angiography was performed and showed an ectopic right coronary artery (RCA) originating from the left cusp. The aberrant RCA

\footnotetext{
From Cardiac Surgery, Erasme Hospital, ${ }^{a}$ Free University of Brussels, Brussels, Belgium, and Cardiac Surgery, Montreal Heart Institute, ${ }^{\mathrm{b}}$ Montreal, Quebec, Canada.

Received for publication Sept 1, 2006; accepted for publication Sept 13, 2006.

Address for reprints: Philippe Demers, MD, Heart Surgery, Heart Institute of Montreal, 5000 rue Bélanger, Montreal, Quebec, Canada (E-mail: chagnondemers@videotron.ca).

J Thorac Cardiovasc Surg 2007;133:258-9

$0022-5223 / \$ 32.00$

Copyright @ 2007 by The American Association for Thoracic Surgery doi:10.1016/j.jtcvs.2006.09.012
}

was ectatic and resulted in an auricular branch with a fistula to the superior vena cava (Figure 1). A computed axial tomography scan confirmed the location of the arteriovenous fistula and showed dilatation of the aortic root and ascending aorta (Figure 2). Dynamic imaging showed compression of the abnormally located RCA between the aortic root aneurysm and the pulmonary artery. Magnetic resonance imaging studies of the abdomen disclosed no other vascular abnormality.

Transesophageal echocardiography was done before the operation, and the arteriovenous fistula was visualized (Figure E1). At the opening of the pericardium, the dilated RCA was identified (Figure E2). The auricular branch of the RCA responsible for arteriovenous fistulization was clipped. Bypass was initiated to perform a Bentall procedure. After aortotomy, 2 ostia were seen in the left sinus, the RCA had a partial intramural course in the aortic wall and was resected, and direct anastomosis of the RCA to the prosthetic tube was performed in the usual anatomic location. The left main artery (LMA) was located at the sinotubular junction and was difficult to mobilize, making direct button anastomosis haz-

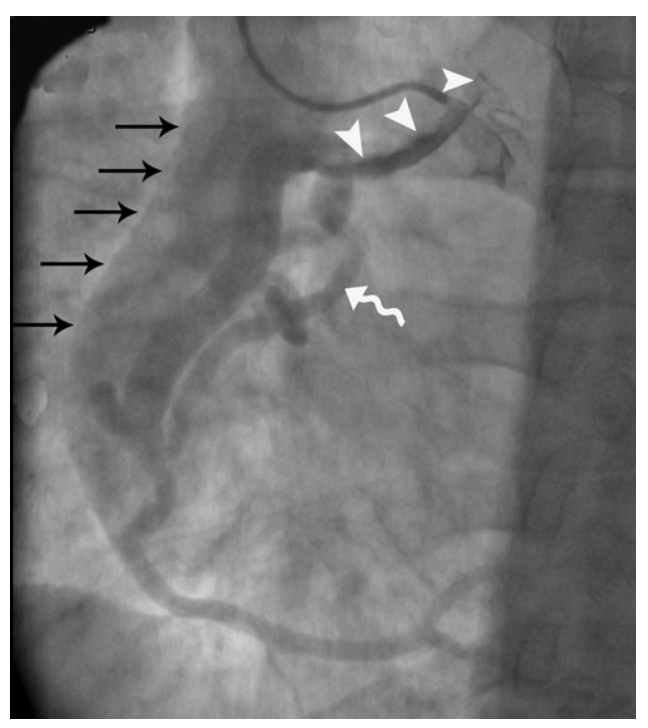

Figure 1. Superior vena cava opacified during right coronary injection (black arrows), right dilated coronary artery (white arrow heads), and auricular branch responsible for the fistula (white wavy arrow). 


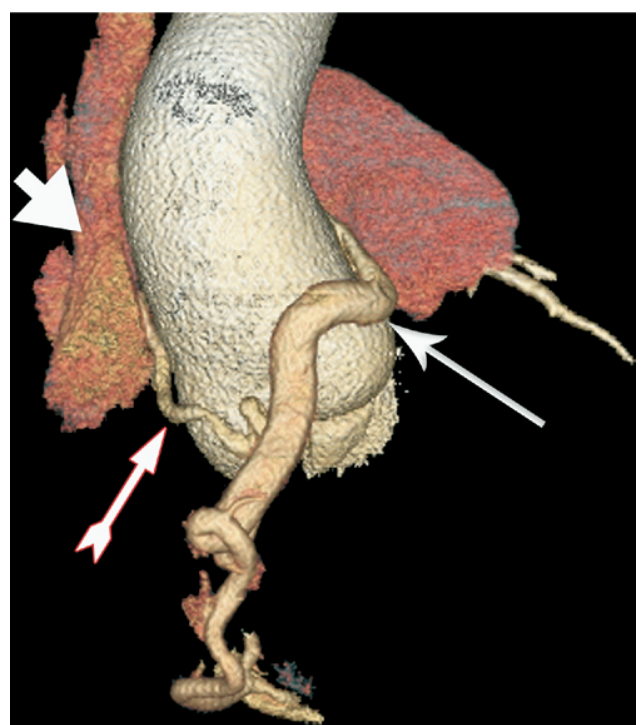

Figure 2. Superior vena cava (large white arrow); branch of the right coronary artery responsible of the fistula (red-white arrow); and right coronary artery with abnormal origination (thin white arrow).

ardous; we choose to interpose a 6-mm Dacron graft between the LMA and the aorta (Figure E3). The postoperative course was uneventful, and on the second day, an echocardiogram showed normalization of right ventricular function. Pathologic examination of the aorta showed typical and extensive cystic medial degeneration of the aortic wall.

\section{Discussion}

Anomalous origination of the RCA from the left cusp has a reported frequency of $0.92 \%$. $^{1}$ The exact clinical relevance of an anomaly is not well defined; the intramural proximal course of the artery might be a weak spot prone to dynamic compression and exercise ischemia. This phenomenon has been exacerbated in our patient by higher wall pressure caused by Laplace's rule because the patient had a dilatation of the aortic root. The patient's annuloaortic ectasia was remarkable because of his young age and the fact that he had no marfanoid features or family history of aortic aneurysm and no other arterial lesion suggestive of connective tissue disease. ${ }^{2}$ Nonetheless, histologic features were those of severe cystic medial degeneration. The third rare anomaly was the RCA fistula to the superior vena cava. Coronary artery fistulas have an incidence of $0.3 \%$ to $0.8 \%$ in angiographic series, ${ }^{3}$ but fistulous drainage in the superior vena cava accounts for only $1 \%$ of these. ${ }^{4}$ Clinical presentation is usually asymptomatic in adulthood, but coronary dilatation, as seen in our patient, is usual and does not depend on the shunt size. ${ }^{5}$ The congenital fistula and the abnormal origination were the substratum for the dynamic RCA compression and right ventricular ischemia that occurred as the aortic root enlarged, bringing the patient to our attention. Surgical alternatives, namely valve-sparing root replacement versus composite valve graft, were presented to the patient, who did not want an aortic valve-sparing procedure because of the small risk of medium-term reoperation. In any case, the abnormal left coronary angulation unrecognized before the intervention would have made the reconstruction unfeasible. Unpublished experience in our institution favors prosthetic grafts over vein grafts to reimplant the LMA. To the best of our knowledge, this is the first report of these 3 rare abnormalities combined in 1 patient.

\section{References}

1. Angelini P. Coronary artery anomalies-current clinical issues: definitions, classification, incidence, clinical relevance, and treatment guidelines. Tex Heart Inst J. 2002;29:271-8.

2. Guo D, Hasham S, Kuang SQ, et al. Familial thoracic aortic aneurysms and dissections: genetic heterogeneity with a major locus mapping to 5q13-14. Circulation. 2001;103:2461-8.

3. Gowda RM, Vasavada BC, Khan IA. Coronary artery fistulas: clinical and therapeutic considerations. Int J Cardiol. 2006;107:7-10.

4. Levin DC, Fellows KE, Abrams HL. Hemodynamically significant primary anomalies of the coronary arteries. Angiographic aspects. Circulation. 1978;58:25-34.

5. Vitarelli A, De CG, Conde Y, et al. Assessment of congenital coronary artery fistulas by transesophageal color Doppler echocardiography. Am J Med. 2002;113:127-33. 


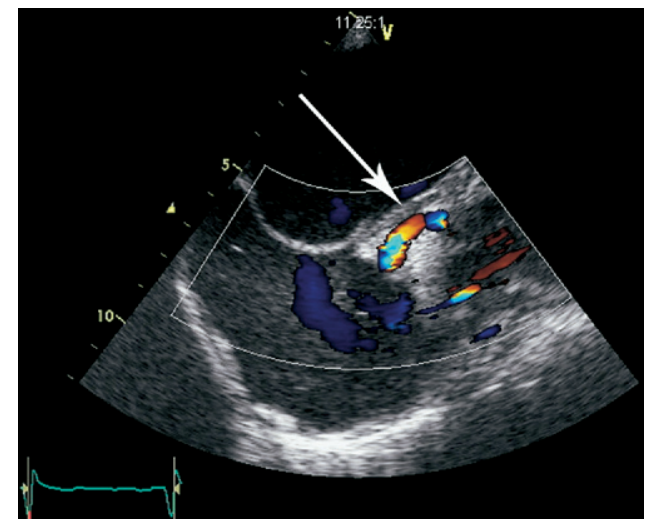

Figure E1. Auricular branch with fistula in the superior vena cava (white arrow).

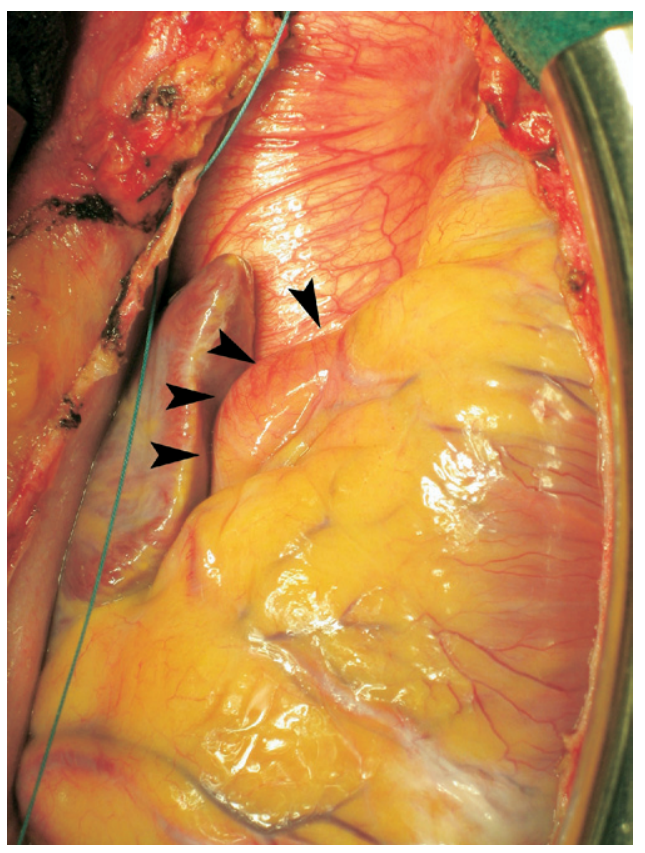

Figure E2. Ectatic right coronary artery (black arrow heads). 


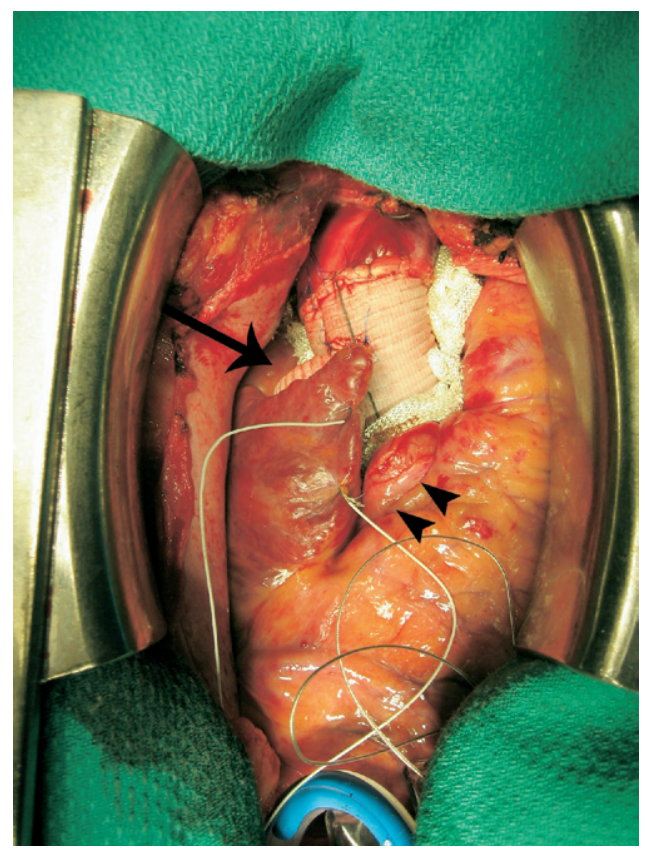

Figure E3. Right ectatic coronary artery (arrow heads); and left coronary artery reimplanted on the anterior portion of the arotic tube via a $6 \mathrm{~mm}$ Dacron (black arrow). 